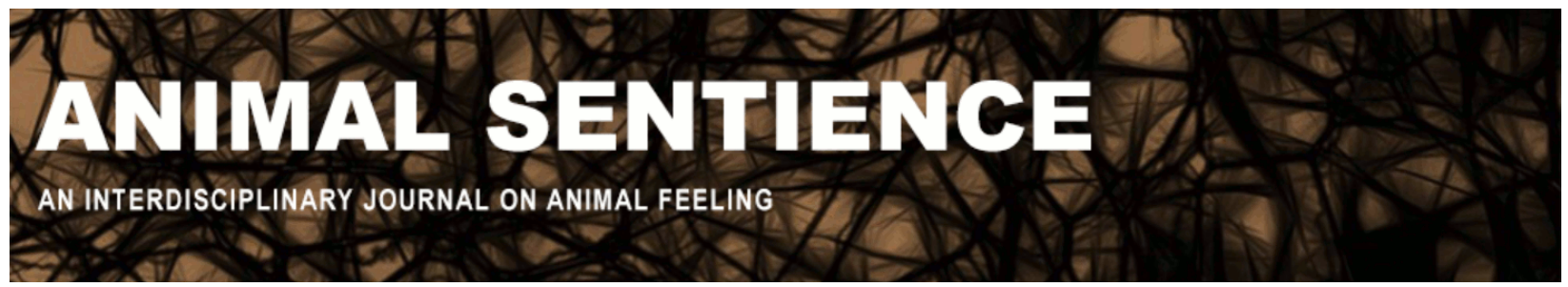

Haikonen, Pentti O. (2016) On the sentience of fish. Animal Sentience 3(8) DOI: $10.51291 / 2377-7478.1035$

Date of submission: $2015-10-03$

Date of acceptance: 2015-12-11

(c) (i)




\title{
On the sentience of fish
}

Commentary on Key on Fish Pain

\author{
Pentti O. Haikonen \\ Department of Philosophy \\ University of Illinois at Springfield
}

\begin{abstract}
Key's (2016) target article, "Why fish do not feel pain," is based on a moralistic fallacy where conclusions about natural conditions are drawn not from research and experiments, but from subjective moral views on how things should be. Moreover, the neurobiological findings purporting to show that fish do not feel pain are insufficient for drawing this conclusion.
\end{abstract}

Pentti O. A. Haikonen pentti.haikonen@pp.inet.fi is Adjunct Professor at University of Illinois at Springfield. He is the author of Consciousness and Robot Sentience: Singapore: World Scientific (2012); Robot Brains; circuits and systems for conscious machines: UK: Wiley (2007); and The Cognitive Approach to Conscious Machines: UK: Imprint Academic (2003).

https://www.youtube.com/user/PenHaiko

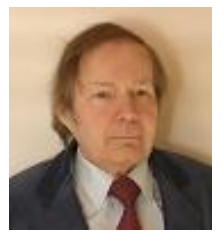

The moralistic fallacy is a faulty form of reasoning where conclusions about natural conditions are drawn not from research and experiment, but from subjective moral views on how things ought to be. A well-known example is the denial of the heliocentric model. "The earth cannot orbit the sun, because this would undermine religion and morals." It should be clear that in this day and age there is no place for this kind of reasoning in scientific research even when the moral conclusions are valid.

Unfortunately, Key's (2016) target article, "Why fish do not feel pain," is based on precisely such reasoning, beginning with a description of the problems that might arise for the fishing industry and the economy in general were it to be shown that fish can feel pain. Key then notes that it would accordingly be very important to show that this is not the case, and then goes on to review some selected neurobiological findings and interprets them as showing that fish do not have the necessary brain structures for feeling pain.

Even if we set the fallacy aside, does Key's line of argument demonstrate that fish cannot feel pain? I am not convinced. He notes that pain is generated by neural processing in the brain and identifies the brain regions that according to him are necessary to feel pain. This list of painrelated brain regions is based on experiments showing neural activity in certain brain regions have been found to be correlated with pain. These areas include prefrontal cortex, anterior cingulate cortex, somatosensory areas SI and SII and the insular cortex. These (and possibly other) brain areas are active during pain, but not selectively so, as they are also active at other times. Key also cites reports where high-frequency EEG gamma oscillations in discrete cortical 
regions are associated with feeling pain and interpreted as possibly encoding pain. From these data he concludes that since the cortex is both necessary and sufficient for the feeling of pain in humans, it is reasonable to infer that fish do not feel pain because their brains lack many of the neuroanatomical features he views as necessary for pain.

This reasoning has serious shortcomings. Key makes the implicit assumption that the neural processes that generate pain require rather large resources and brain areas. Obviously pain is in the brain and obviously pain-related activity in various parts (if not all) of the brain including the cortex can be detected by various methods. But these findings do not show that the activity detected during pain is actually generating the feeling of pain. These regions are also active during pain-free conditions; it is hence possible that these various regions just produce cognitive processing (Haikonen 2003, 2012) and other responses to pain whereas the actual feeling of pain is generated elsewhere and by other means. The neural mechanism that actually generates the feeling of pain is not yet known; thus, we do not know whether it is a simple one that can fit into the diminutive fish brain or a complex one calling for large cortical resources. The fact that we do not currently understand pain does not imply that it requires great neural complexity.

The conclusion that the brain regions identified by Key are necessary for feeling pain is hence premature. The comparison of the fish brain and the human brain fails, as does Key's ultimate conclusion that fish do not feel pain because their brain lacks the required neuroanatomical features of the human brain.

\section{References}

Haikonen, P. O. (2003). The Cognitive Approach to Conscious Machines. UK: Imprint Academic.

Haikonen, P. O. (2012). Consciousness and Robot Sentience. Singapore: World Scientific.

Key, B. (2016). Why fish do not feel pain. Animal Sentience 2016.3. 